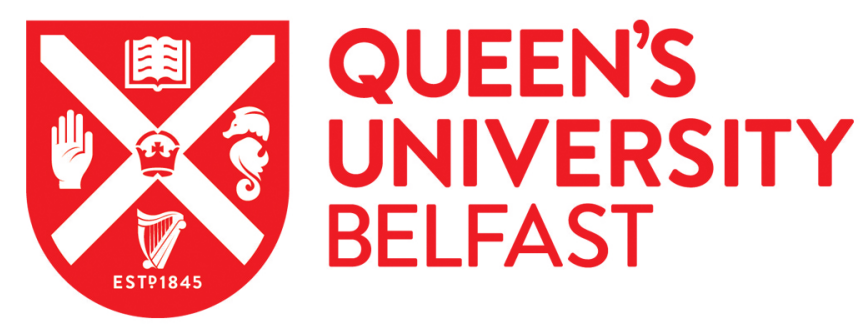

\title{
Dynamics of atomic spin-orbit-state wave packets produced by short- pulse laser photodetachment
}

Gribakin, G. F., \& Law, S. M. K. (2016). Dynamics of atomic spin-orbit-state wave packets produced by shortpulse laser photodetachment. Physical Review A (Atomic, Molecular, and Optical Physics), 94(5), [053402]. https://doi.org/10.1103/PhysRevA.94.053402

Published in:

Physical Review A (Atomic, Molecular, and Optical Physics)

Document Version:

Publisher's PDF, also known as Version of record

Queen's University Belfast - Research Portal:

Link to publication record in Queen's University Belfast Research Portal

Publisher rights

(C) 2016 American Physical Society.

This work is made available online in accordance with the publisher's policies. Please refer to any applicable terms of use of the publisher.

\section{General rights}

Copyright for the publications made accessible via the Queen's University Belfast Research Portal is retained by the author(s) and / or other copyright owners and it is a condition of accessing these publications that users recognise and abide by the legal requirements associated with these rights.

Take down policy

The Research Portal is Queen's institutional repository that provides access to Queen's research output. Every effort has been made to ensure that content in the Research Portal does not infringe any person's rights, or applicable UK laws. If you discover content in the Research Portal that you believe breaches copyright or violates any law, please contact openaccess@qub.ac.uk. 


\title{
Dynamics of atomic spin-orbit-state wave packets produced by short-pulse laser photodetachment
}

\author{
S. M. K. Law and G. F. Gribakin* \\ Center for Theoretical Atomic, Molecular and Optical Physics, School of Mathematics and Physics, Queen's University Belfast, \\ Belfast BT7 1NN, United Kingdom
}

(Received 24 August 2016; published 2 November 2016)

\begin{abstract}
We analyze the experiment by Hultgren et al. [Phys. Rev. A 87, 031404 (2013)] on orbital alignment and quantum beats in coherently excited atomic fine-structure manifolds produced by short-pulse laser photodetachment of $\mathrm{C}^{-}, \mathrm{Si}^{-}$, and $\mathrm{Ge}^{-}$negative ions, and derive a formula that describes the beats. Analysis of the experimental data enables us to extract the noncoherent background contribution for each species, and indicates the need for a full density-matrix treatment of the problem.
\end{abstract}

DOI: 10.1103/PhysRevA.94.053402

\section{INTRODUCTION}

In this paper we analyze the dynamics of quantum wave packets produced by coherent excitation of atomic finestructure manifolds in laser photodetachment experiments, and probed by multiphoton ionization. We show that for atoms with $n p^{2}{ }^{3} P$ ground state the corresponding signal has a very specific shape, which we determine analytically and find to be in good agreement with experiment.

The development of laser pulses of few-femtosecond duration allows one to resolve the electron motion in valence shells of atoms and molecules in the time domain (see, e.g., Refs. [1-6]). Recently 100 fs pulse pump-probe experiments $[7,8]$ were carried out to investigate the dynamics induced by the spin-orbit interaction in neutral atoms. In these experiments $\mathrm{C}, \mathrm{Si}$, and $\mathrm{Ge}$ atoms with outer $n p^{2}$ configuration were prepared in the ${ }^{3} P$ ground state by photodetachment of the respective half-filled valence shell negative ions $\left(n p^{3}{ }^{4} S\right)$ by a linearly polarized pump pulse. Upon interaction with the infrared laser pulse, the emission of $p$ electrons with orbital angular momentum projection $m=0$ is strongly favored in comparison to $m= \pm 1$. This causes the formation of a state with an electron density hole localized along the pump laser polarization axis and constitutes the orbital alignment effect. Such a state is not an eigenstate of the atomic Hamiltonian when the spin-orbit interaction is included, but a superposition of the fine-structure levels ${ }^{3} P_{J}$, which evolves in time according to the energy splittings in this manifold. This means that even for a light atom, such as $\mathrm{C}$, the relativistic spin-orbit interaction is essential in determining the electron dynamics following the pump pulse. In Refs. [7,8] this effect was probed by applying a time-delayed ionizing probe pulse and measuring the signal of ionized electrons for parallel and perpendicular polarization of the pump and probe pulses, as a function of the time delay.

The experimental findings demonstrated the dependence of the ionization yield on the time-varying hole density and the presence of quantum beat oscillations of the signal with the delay time for $\mathrm{C}$ and $\mathrm{Si}$ (with no distinct signal for $\mathrm{Ge}$ ). This showed that electron dynamics resulting from the spin-orbit interaction could be observed for both lighter and heavier

\footnotetext{
*g.gribakin@qub.ac.uk
}

atoms. In a recent paper [9] Rey and van der Hart used $R$-matrix theory with time dependence (RMT) to model the experiment of Refs. [7,8]. They calculated the electron spectra following ionization of carbon in the initial orbitally aligned states with magnetic quantum numbers $M_{L}=0$ and 1 , and observed significant differences between these two cases, matching the experimental findings. They also considered the evolution of the fine-structure-state wave packet with the pump-probe delay time and simulated the experimental signal by integrating electron emission within the cone of 11.7 degrees around the polarization direction of the probe pulse with momenta $p \geqslant 0.4$ a.u. The scaled normalized yield obtained in this way was found to be in good agreement with the experimental data from Ref. [8].

In the present work we show that the experimental results can be described in a much simpler manner. We use the assumption (similar to that used in Ref. [9] and key to the experimental method of Refs. [7,8]) that removal of $m=0$ electrons dominates both the photodetachment (pump) and subsequent photoionization (probe), and consider the motion of the $n p^{2}{ }^{3} P$ fine-structure wave packet in $\mathrm{C}, \mathrm{Si}$, or $\mathrm{Ge}$. This gives a simple analytical expression for the signal as a function of the pump-probe time delay. For pulses whose duration is short compared with the beat periods of the wave packet, the laser pulse characteristics (i.e., their intensity, wavelength, etc.) and the dynamics of their interaction with the ion and the neutral atom play no essential role in the process. In order to make comparisons with experimental results of Ref. [7] we scale the signal to account for the background counts that could be present under the experimental conditions. This allows us to analyze the contribution of background to the observed signal beats and to effectively describe the loss of coherence in the wave packets for systems in which the duration of the laser pulses is comparable to or greater than the beat periods. (A proper treatment of the latter case calls for numerical calculations of the laser photoionization dynamics and computation of the time-dependent density matrix of the fine-structure manifold.)

The structure of the paper is as follows. In Sec. II we derive the expression that describes the beats of the signal due to the time evolution of the atomic-state wave packet. In Sec. III we use our analytical expression to model the experimental data and compare with the phenomenological simulation used in Refs. [7,8]. Section IV provides brief conclusions. Note that we use atomic units throughout. 


\section{THEORY}

Before the arrival of the pump pulse the negative ions are in the $n p^{3}{ }^{4} S$ ground state with the total orbital angular momentum $L=0$ and spin $S=3 / 2$, and their projections $M_{L}=0$ and $M_{S}=-3 / 2, \ldots, 3 / 2$. The total angular momentum and its projection are $J=S$ and $M=M_{S}$.

We assume that after an instantaneous photodetachment of an electron with $m=0$, the atoms are produced in a $n p^{2}{ }^{3} P$ state at zero time delay, after which this state evolves according to the energy splittings of the fine-structure manifold. The initial state of the atom is described by its total orbital and spin angular momentum quantum numbers $L$ and $S$ with projections $M_{L}$ and $M_{S}$, respectively, which we denote by $\left|L, M_{L} ; S, M_{S}\right\rangle$ (or a superposition of such states, see below). In the $L S$-coupling scheme, the time evolution of the initial atomic state $\left|L, M_{L} ; S, M_{S}\right\rangle$ is given by

$$
|\Psi(t)\rangle=\sum_{J} e^{-i E_{J} t} C_{L M_{L} S M_{S}}^{J M}|J, M\rangle
$$

where $|J, M\rangle$ is the fine-structure energy eigenstate with the total angular momentum $J$, projection $M=M_{L}+M_{S}$, and energy $E_{J}, C_{L M_{L} S M_{S}}^{J M}$ denotes a Clebsch-Gordan coefficient, and $|\Psi(0)\rangle=\left|L, M_{L} ; S, M_{S}\right\rangle$.

To find the occupancies of the electron orbitals with $m=0$ in $|\Psi(t)\rangle$, which determine the ionization signal after the probe pulse, we expand the fine-structure states $|J, M\rangle$ in the basis of $L S$ states $\left|L, M_{L} ; S, M_{S}\right\rangle$ (see the Appendix for the explicit form of these in terms of the single-particle states),

$$
|\Psi(t)\rangle=\sum_{J} \sum_{M_{L}^{\prime}, M_{S}^{\prime}} e^{-i E_{J} t} C_{L M_{L} S M_{S}}^{J M} C_{L M_{L}^{\prime} S M_{S}^{\prime}}^{J M}\left|L, M_{L}^{\prime} ; S, M_{S}^{\prime}\right\rangle,
$$

where the second sum is over all $M_{L}^{\prime}$ and $M_{S}^{\prime}$ such that $M_{L}^{\prime}+$ $M_{S}^{\prime}=M$.

The removal of an $m=0$ electron from the initial $M_{S}=$ $3 / 2$ anion state produces the atomic state $|1,0 ; 1,1\rangle$. The subsequent time evolution of the atomic wave packet is found by applying Eq. (2) and evaluating the appropriate Clebsch-Gordan coefficients,

$$
\begin{aligned}
\left|\Psi_{1}(t)\right\rangle= & \frac{1}{2}\left(e^{-i E_{2} t}+e^{-i E_{1} t}\right)|1,0 ; 1,1\rangle \\
& +\frac{1}{2}\left(e^{-i E_{2} t}-e^{-i E_{1} t}\right)|1,1 ; 1,0\rangle .
\end{aligned}
$$

The corresponding ionization signal after the probe pulse is proportional to the probability of finding an $m=0$ electron in the state (3),

$$
\begin{aligned}
& S_{\|}^{(1)}=\frac{1}{2}\left(1-\cos \omega_{21} t\right), \\
& S_{\perp}^{(1)}=\frac{1}{4}\left(3+\cos \omega_{21} t\right),
\end{aligned}
$$

for the parallel and perpendicular polarization of the probe, respectively. Here $\omega_{J J^{\prime}}=E_{J}-E_{J^{\prime}}$, and the explicit forms of the atomic states given in the Appendix were used.

The removal of an $m=0$ electron from the initial $M_{S}=$ $1 / 2$ anion state produces a superposition of atomic states,

$$
\frac{1}{\sqrt{3}}|1,0 ; 1,1\rangle+\sqrt{\frac{2}{3}}|1,0 ; 1,0\rangle,
$$

(see the Appendix), whose time evolution is given by

$$
\begin{aligned}
\left|\Psi_{2}(t)\right\rangle= & \frac{1}{\sqrt{3}}\left|\Psi_{1}(t)\right\rangle+\sqrt{\frac{2}{3}}\left(\frac{2}{3} e^{-i E_{2} t}+\frac{1}{3} e^{-i E_{0} t}\right)|1,0 ; 1,0\rangle \\
& +\sqrt{\frac{2}{3}}\left(\frac{1}{3} e^{-i E_{2} t}-\frac{1}{3} e^{-i E_{0} t}\right)(|1,1 ; 1,-1\rangle \\
& +|1,-1 ; 1,1\rangle) .
\end{aligned}
$$

This gives the probabilities of finding an $m=0$ electron at time $t$ as

$$
\begin{gathered}
S_{\|}^{(2)}=\frac{1}{6}\left(1-\cos \omega_{21} t\right)+\frac{8}{27}\left(1-\cos \omega_{20} t\right), \\
S_{\perp}^{(2)}=\frac{1}{12}\left(7+\cos \omega_{21} t\right)+\frac{1}{27}\left(5+4 \cos \omega_{20} t\right) .
\end{gathered}
$$

Note that since the Clebsch-Gordan coefficient $C_{1010}^{10}$ is zero, no interference is observed between the $J=0$ and $J=1$ sublevels. This is in agreement with the experimental analysis [7,8], which allowed for the presence of the $\omega_{10}=E_{1}-E_{0}$ beat frequency, but found its contribution statistically insignificant. Note also that the sum $S_{\|}^{(i)}+2 S_{\perp}^{(i)}=2$, independently of time, which could be expected since there are two orthogonal directions perpendicular to the polarization of the pump pulse.

The total signals for the parallel and perpendicular polarizations of the pump and probe pulses are proportional to $S_{\|}=S_{\|}^{(1)}+S_{\|}^{(2)}$ and $S_{\perp}=S_{\perp}^{(1)}+S_{\perp}^{(2)}$. (The initial anion states with $M_{S}=-3 / 2$ and $-1 / 2$ give the identical contribution.) The normalized electron yield measured in the experiment is

$$
S(t)=\frac{S_{\perp}-S_{\|}}{S_{\perp}+S_{\|}}
$$

Using Eqs. (4), (5), (8), and (9), and allowing for a constant time shift $t_{0}$ related to the uncertainty of the zero time delay [8], and for some signal background that may contribute to $S_{\|}$ and $S_{\perp}$, we obtain

$$
S(t)=\frac{\frac{5}{9}+\cos \left[\omega_{21}\left(t-t_{0}\right)\right]+\frac{4}{9} \cos \left[\omega_{20}\left(t-t_{0}\right)\right]+\Delta S_{b}}{\frac{67}{27}-\frac{1}{3} \cos \left[\omega_{21}\left(t-t_{0}\right)\right]-\frac{4}{27} \cos \left[\omega_{20}\left(t-t_{0}\right)\right]+S_{b}},
$$

where $S_{b}$ and $\Delta S_{b}$ are the sum and difference of the background contributions for the parallel and perpendicular polarizations. Such background can also account for reduced coherence of the wave packet when the pump pulse duration becomes comparable to or greater than the beat periods (see Sec. III). Note that a better quantity than that in Eq. (10) would probably be the ratio $\left(2 S_{\perp}-S_{\|}\right) /\left(2 S_{\perp}+S_{\|}\right)$, in which the denominator should be constant.

The contribution of the oscillating terms in the denominator of Eq. (11) is relatively small, even in the absence of any background $S_{b}$. This means that $S(t)$ is close to a simple linear combination of a constant and two beat components with frequencies $\omega_{21}$ and $\omega_{20}$. Equation (11) shows that the relative contribution of the beats with frequencies $\omega_{21}$ and $\omega_{20}$ is fixed, and the beat period between the higher-lying levels $J=1,2$ gives the dominant contribution. Note also that Eq. (11) with $\Delta S_{b}=S_{b}=0$ predicts a positive constant offset $\bar{S}=15 / 67 \sim 0.2$, which is qualitatively similar to the observations (see Sec. III). 

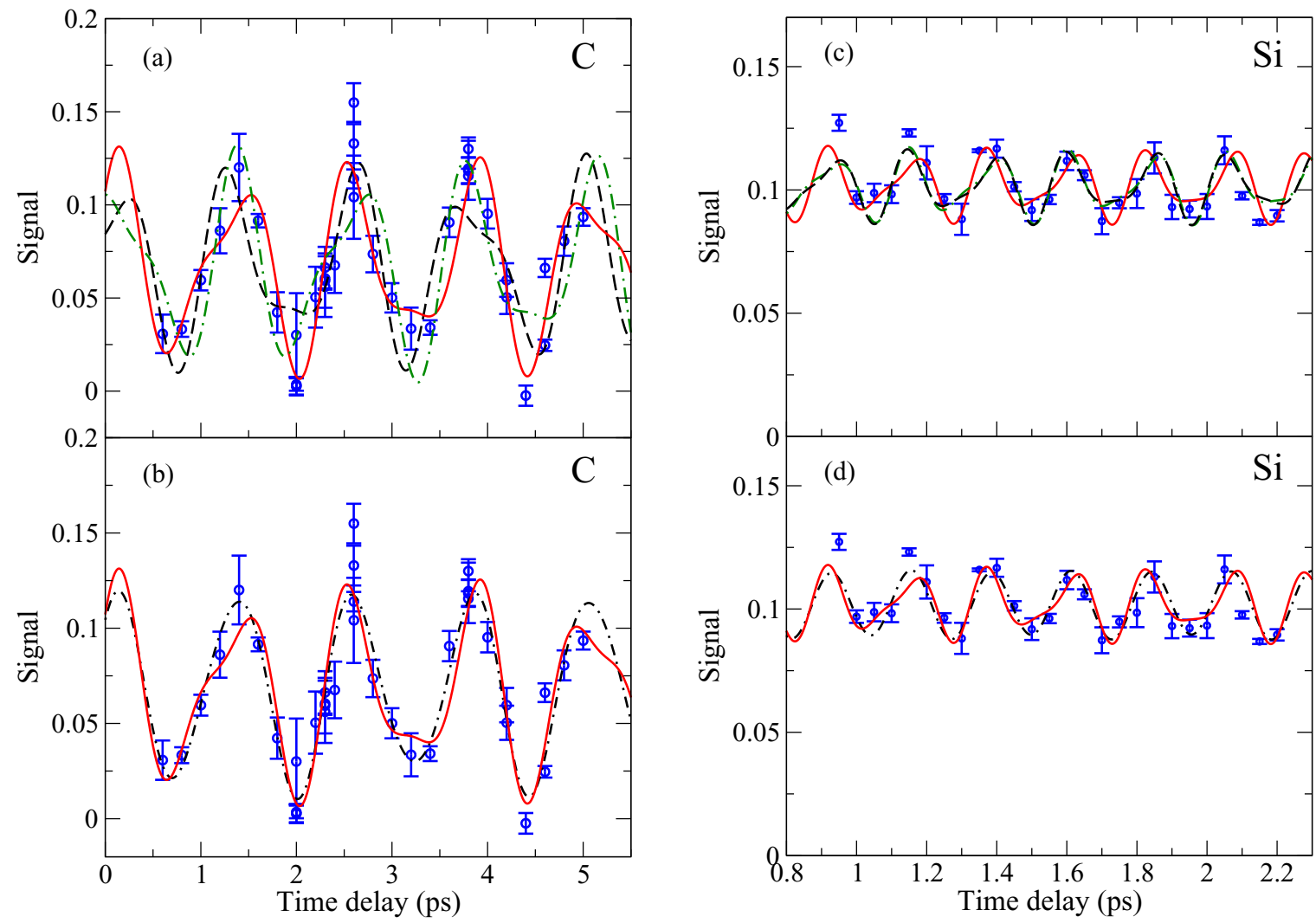

FIG. 1. Normalized electron ionization yield as a function of time delay for C [(a) and (b)] and Si [(c) and (d)]. The graphs in (a) and (c) show several theoretical fits using Eq. (11) with different time shift parameter $t_{0}$ that best model the experimental data [8] plotted in blue circles in each panel. The three fits for $\mathrm{C}$ in (a) correspond to $t_{0}=-1.127$ ps (black dashed line), 0.144 ps (red solid line), and $1.381 \mathrm{ps}$ (green dash-dotted line). For $\mathrm{Si}$ in (c) the fits correspond to $t_{0}=-0.213 \mathrm{ps}$ (black dashed line), 0.013 ps (red solid line), and $0.244 \mathrm{ps}$ (green dash-dotted line). Other parameters are listed in Table I. (b) and (d) compare the best fit from (a) and (c) for C and Si, respectively (red solid line) with the fit obtained by using Eq. (12) [7] (black dash-dotted line).

In analyzing the experimental data, the authors of Refs. [7,8] used the following phenomenological function

$$
\begin{aligned}
f(t)= & c_{0}+\alpha_{1} \cos \left[\omega_{21}\left(t-t_{0}\right)\right]+\alpha_{2} \cos \left[\omega_{20}\left(t-t_{0}\right)\right] \\
& +\alpha_{3} \cos \left[\omega_{10}\left(t-t_{0}\right)\right],
\end{aligned}
$$

with five fitting parameters: a constant offset $c_{0}$, amplitudes $\alpha_{i}$ $(i=1,2,3)$ of all three possible beats with frequencies $\omega_{21}$, $\omega_{20}$, and $\omega_{10}$, and $t_{0}$.

The beat frequencies are determined by the corresponding energy splittings [10]: $\omega_{21}=5.086,27.510$, and $160.65 \mathrm{ps}^{-1}$, $\omega_{20}=8.175,42.028$, and $265.56 \mathrm{ps}^{-1}$, and $\omega_{10}=3.089$, 14.524 , and $104.95 \mathrm{ps}^{-1}$, for $\mathrm{C}, \mathrm{Si}$, and $\mathrm{Ge}$, respectively. The corresponding beat periods are $\tau_{J J^{\prime}}=2 \pi / \omega_{J J^{\prime}}$. In Sec. III we compare the results obtained using our three-parameter fits (11) with those of Eq. (12).

\section{RESULTS AND DISCUSSION}

Figure 1 displays the results for the normalized yield $S(t)$, Eq. (10), as a function of time delay for carbon [Figs. 1(a) and 1(b)] and silicon [Figs. 1(c) and 1(d)]. The experimental results from Ref. [8], obtained from momentum-resolved images for high-energy ( $p \geqslant 0.4$ a.u.) ionized electrons, are shown by blue circles in each panel. They are compared with (i) our analytical formula (11) fitted using $t_{0}, S_{b}$, and $\Delta S_{b}$ as free parameters, and (ii) the phenomenological five-parameter fit Eq. (12) used previously in Refs. [7,8]. By varying the range of the time shift parameter $t_{0}$ in Eq. (11), several locally optimal fits may be achieved, the one with the smallest absolute value of $t_{0}$ being the overall best (shown by the solid red line). Figure 2 shows three fits for Ge, using only Eq. (11) and plotted similarly to Figs. 1(a) and 1(c). A full list of fitted parameter values is in Table I.

Simulation of the experimental data by means of Eq. (11) for $\mathrm{C}$ and $\mathrm{Si}$ clearly shows the temporal oscillations originating from quantum beat interference between the coherently populated $J$ sublevels of the ${ }^{3} P$ ground state. These oscillations are faster for heavier atoms, as observed in the experiment, which is directly linked to the decrease in the spin-orbit periods $\tau_{J J^{\prime}}$ for larger fine-structure splitting energies $\omega_{J J^{\prime}}$. As predicted by Eq. (11), the observed beat periods are dominated by the $J=1,2$ sublevel contributions with $\tau_{21}=1.24 \mathrm{ps}, \tau_{21}=$ 0.23 ps and $\tau_{21}=39.11 \mathrm{fs}$ for $\mathrm{C}, \mathrm{Si}$, and $\mathrm{Ge}$, respectively.

By introducing the appropriate background parameters in the fits, good agreement with the experimental data is observed in Fig. 1 for $\mathrm{C}$ and $\mathrm{Si}$. The best fits [shown by solid red curves in Figs. 1(b) and 1(d)] correspond to the smallest absolute values of $t_{0}(\lesssim 100 \mathrm{fs})$, other $t_{0}$ values differing from it by $\sim \tau_{21}$. For both atoms, the beat pattern is dominated by the $\tau_{21}$ period component, with the $\tau_{20}$ beat component producing 


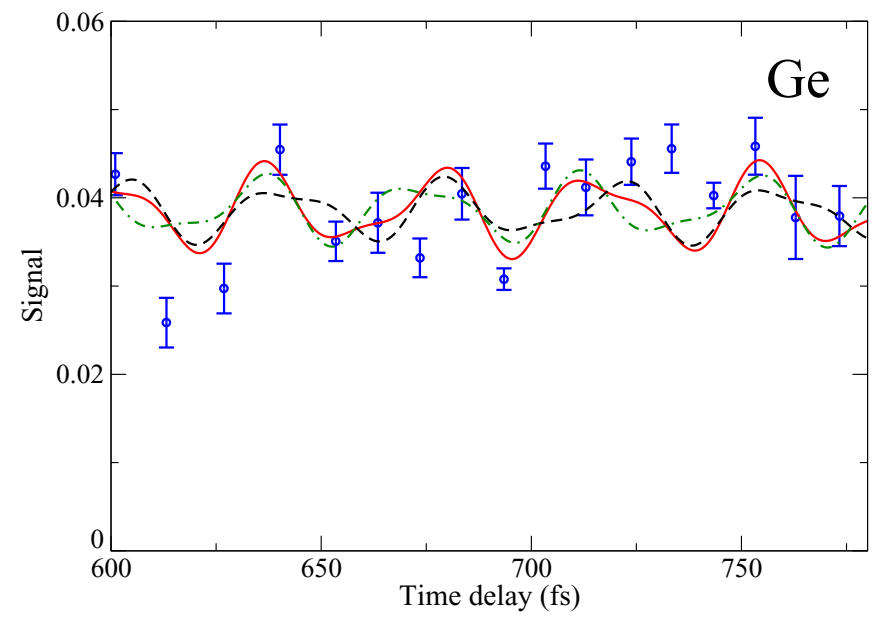

FIG. 2. Normalized electron yield as a function of time delay for Ge. Experimental data [8] (blue circles); fits using Eq. (11) correspond to $t_{0}=-27.10 \mathrm{fs}$ (solid red line), $15.66 \mathrm{fs}$ (dashed black line), and 47.98 fs (dash-dotted green line); other parameters are in Table I.

a characteristic knee visible at even half-periods. A similar pattern was observed in numerical simulations for $\mathrm{C}$ by Rey and van der Hart [9], but it is totally absent from the fit with the function $f(t)$, Eq. (12), used in the experimental papers [7,8] [black dash-dotted lines in Figs. 1(b) and 1(d)].

For Ge, however, the oscillatory behavior predicted by Eq. (11) does not provide a good description of the experimental data for any choice of parameters (see Fig. 2). A related feature of the data is that the scale of the oscillations becomes very small in Ge compared with $\mathrm{C}$ and $\mathrm{Si}$. This can be seen from the fitted values of $S_{b}$ and $\Delta S_{b}$ in Table I. From Eq. (11), the time-independent part of the asymmetry is determined by the ratio $\bar{S}=\left(5 / 9+\Delta S_{b}\right) /\left(67 / 27+S_{b}\right) \sim \Delta S_{b} / S_{b}$, while the amplitude of the beats is $\sim 1 / S_{b}$. The data in Figs. 1 and 2 , and in Table I show that $\Delta S_{b} / S_{b} \sim 0.05-0.1$ for all three species, while the amplitude of the beats decreases from 0.05 for $\mathrm{C}$, to 0.01 for $\mathrm{Si}$, and $4 \times 10^{-3}$ for $\mathrm{Ge}$. The latter value is close to the size of error bars in the experimental data for Ge.

TABLE I. Values of parameters $t_{0}, \Delta S_{b}$, and $S_{b}$ used in Eq. (11) to fit the experimental data from Ref. [8] in Figs. 1 and 2. The $\chi^{2}$ error representing the quality of the fit for each set of parameters is also shown. The choice of parameters that gives the best fit for each atom is shown in bold.

\begin{tabular}{lrrrc}
\hline \hline & \multicolumn{3}{c}{ Best-fit parameters } & \multicolumn{1}{c}{ Error } \\
\cline { 2 - 4 } Atom & \multicolumn{1}{c}{$\Delta S_{b}$} & \multicolumn{1}{c}{$S_{b}$} & \multicolumn{1}{c}{$t_{0}(\mathrm{ps})$} & $\chi^{2}$ \\
\hline $\mathrm{C}$ & 1.11 & 22.30 & -1.127 & $1.98 \times 10^{-2}$ \\
& $\mathbf{1 . 0 2}$ & $\mathbf{2 0 . 9 6}$ & $\mathbf{0 . 1 4 4}$ & $\mathbf{8 . 8 9} \times \mathbf{1 0}^{-\mathbf{3}}$ \\
& 0.97 & 20.45 & 1.381 & $1.67 \times 10^{-2}$ \\
$\mathrm{Si}$ & 8.88 & 90.38 & -0.213 & $1.39 \times 10^{-3}$ \\
& $\mathbf{8 . 7 7}$ & $\mathbf{8 8 . 8 8}$ & $\mathbf{0 . 0 1 3}$ & $\mathbf{1 . 3 5} \times \mathbf{1 0}^{-\mathbf{3}}$ \\
& 8.85 & 89.96 & 0.244 & $1.38 \times 10^{-3}$ \\
$\mathrm{Ge}$ & $\mathbf{9 . 4 0}$ & $\mathbf{2 5 5 . 1 8}$ & $\mathbf{- 0 . 0 2 7}$ & $\mathbf{4 . 3 3} \times \mathbf{1 0}^{-\mathbf{4}}$ \\
& 13.51 & 362.73 & 0.016 & $5.13 \times 10^{-4}$ \\
& 12.10 & 324.13 & 0.048 & $4.79 \times 10^{-4}$ \\
\hline \hline
\end{tabular}

This behavior is related to the effect of the pulse duration in comparison with the beat periods. In the derivation of Eq. (11), the removal of $m=0$ electrons was assumed to be instantaneous, leading to fully coherent (pure) time-dependent states with wave functions (3) and (7). In the experiment $[7,8]$ the duration of the pump and probe pulses was $100 \mathrm{fs}$, which is much shorter the main beat period for $\mathrm{C}$ and shorter than that for $\mathrm{Si}$, but is 2.5 times greater than $\tau_{21}$ for Ge. As a result, the degree of coherence in the spin-orbit wave packet is largest for $\mathrm{C}$, but becomes progressively smaller in $\mathrm{Si}$ and $\mathrm{Ge}$. This results in the reduction of the coherent (oscillatory) part of the signal, with the atomic states produced by the pump becoming closer to a classical ensemble rather than a quantum superposition.

The spin-orbit wave packet in Ge (and to a lesser extent, in $\mathrm{Si}$ ) is also affected by strong dependence of the multiphoton detachment rates on the threshold energy. This leads to a greater suppression of the detachment probability for higherlying final atomic states with $J=1$ and 2, compared with that for the $J=0$ ground state. The magnitudes of the lowest $(J=$ 0 ) and highest $(J=2)$ thresholds are 1.2621 and $1.2675 \mathrm{eV}$ in $\mathrm{C}^{-}, 1.3895$ and $1.4172 \mathrm{eV}^{-1} \mathrm{Si}^{-}$, and 1.2327 and $1.4075 \mathrm{eV}$ in $\mathrm{Ge}^{-}$. Using the method of Ref. [11], we estimate that for a laser pulse with wavelength $\lambda=2055 \mathrm{~nm}$ (as in Refs. $[7,8]$ ) and intensity $I=2 \times 10^{12} \mathrm{~W} / \mathrm{cm}^{2}$ (for which the total detachment probability over 100 fs is close to unity), the increase in the threshold energy from the $J=0$ to $J=2$ state leads to $2 \%, 7 \%$, and $40 \%$ reduction of the detachment rate, for $\mathrm{C}, \mathrm{Si}$, and $\mathrm{Ge}$, respectively. As a result, the contribution of the $J=2$ state to the wave packet (1), which is critical for the magnitude of the beats, can be reduced below the values predicted by the $L S$-coupling coefficients.

The above analysis makes it clear that a complete description of the beat character and spin-orbit coherences of the atomic ensemble requires a density matrix consideration of the problem $[2,5,12]$. Depending on the pump pulse length, strongfield detachment may not generally produce perfectly coherent aligned states. The elements of the density matrix in the $|J, M\rangle$ basis can be determined by calculating the detachment amplitudes for a variety of pulse lengths using existing theory of strong-field photodetachment (e.g., Keldysh-type theory $[11,13-16])$. In this approach the diagonal elements will represent populations of different atomic fine-structure levels for a given $M$, and the magnitude of the off-diagonal elements will describe coherences between the $J$ states. The degree of coherence is then described by the ratio of the off-diagonal elements to the geometric mean of the corresponding diagonal elements. Calculations for the halogen negative ions, whose detachment leads to two fine-structure atomic states, show that the degree of coherence is a function of the ratio $\tau_{p} / \tau_{J J^{\prime}}$, where $\tau_{p}$ is the laser pulse length [12]. For $\tau_{p} / \tau_{J J^{\prime}} \ll 1$ the degree of coherence is close to unity, but it drops quickly for $\tau_{p} / \tau_{J J^{\prime}} \sim 1$ and reaches few-percent values for $\tau_{p} \approx 2 \tau_{J J^{\prime}}$, which is similar to the situation in Ge.

\section{CONCLUSIONS}

We have investigated the evolution of the ground-state spin-orbit wave packets in carbon, silicon, and germanium atoms produced by detachment of $m=0$ electrons from half-filled valence $n p^{3}$ negative ions. A simple analytical 
formula that describes the time-changing alignment of electron orbitals, as probed in the pump-probe experiment, has been derived and applied to the analysis of experimental data $[7,8]$. For $\mathrm{C}$ and $\mathrm{Si}$ the theory provides a good description of temporal beat oscillations, which demonstrate the existence of a coherent superposition of the fine-structure sublevels of the atomic triplet state. The sharp suppression of the coherence degree observed experimentally for Ge demonstrates that the assumption of an instantaneous pulse is insufficient for atoms with shorter beat periods (in the femtosecond range). This calls for a full density-matrix consideration of the problem that would provide a complete description of partially coherent dynamics occurring in spin-orbit manifolds of general atoms with $l \geqslant 1$ valence-electron orbitals.

\section{ACKNOWLEDGMENTS}

The work of S.M.K.L. has been supported by the Department for Employment and Learning, Northern Ireland. We thank I. Kiyan and M. Eklund for providing experimental data and for useful discussions.

\section{APPENDIX: VALENCE-ELECTRON STATES OF ANIONS AND ATOMS OF C, SI, AND GE}

Using the notation $\left|L, M_{L} ; S, M_{S}\right\rangle$ for the state of the atomic system with the total orbital angular momentum $L$ and spin $S$, with $z$ projections $M_{L}$ and $M_{S}$, respectively, the possible initial states of the $n p^{3}{ }^{4} S$ negative ion are

$$
\begin{aligned}
& |0,0 ; 3 / 2,3 / 2\rangle=\begin{array}{|l|l|l|}
\hline \uparrow & \uparrow & \uparrow \\
\hline
\end{array} \\
& |0,0 ; 3 / 2,1 / 2\rangle=\frac{1}{\sqrt{3}}\left(\begin{array}{|l|l|l|}
\hline \uparrow & \uparrow & \downarrow \\
\hline
\end{array}\right. \\
& +\begin{array}{|l|l|l|}
\hline \downarrow & \uparrow & \uparrow \\
\hline
\end{array}
\end{aligned}
$$

where each of the boxes represents a state of three electrons in the $n p$ orbital, with magnetic quantum numbers $m=-1$, 0 , and 1 , and up $(\uparrow)$ or down $(\downarrow)$ spins. The states with $M_{S}=-1 / 2$ and $-3 / 2$ are similar and, owing to the symmetry with respect to reflection in the $x-y$ plane, they need not be considered.
The two-electron $n p^{2}{ }^{3} P$ states of the neutral atom that can be formed by removal of an $m=0$ electron from the above states are

$$
\begin{gathered}
|1,0 ; 1,1\rangle=\begin{array}{|l|l|l|}
\hline \uparrow & & \uparrow \\
\hline
\end{array} \\
|1,0 ; 1,0\rangle=\frac{1}{\sqrt{2}}\left(\begin{array}{|l|l|l|l|l|l|}
\hline \uparrow & & \downarrow \\
\hline
\end{array}\right.
\end{gathered}
$$

or their superposition. Other atomic states that appear in the $L S$ expansion of the fine-structure levels $|J, M\rangle$ linked to the states (A3) and (A4), are

$$
\begin{aligned}
& |1,1 ; 1,0\rangle=\frac{1}{\sqrt{2}}\left(\begin{array}{|l|l|l|}
\hline & \uparrow & \downarrow \\
\hline
\end{array}\right. \\
& |1,-1 ; 1,1\rangle=\begin{array}{|l|l|l|}
\hline \uparrow & \uparrow & \\
\hline
\end{array} \\
& |1,1 ; 1,-1\rangle=\begin{array}{|l|l|l|}
\hline & \downarrow & \downarrow \\
\hline
\end{array}
\end{aligned}
$$

Using states (A3)-(A7) it is straightforward to work out the relative probabilities of removing $m=0$ electron by the ionizing probe pulse with polarization parallel to the pump pulse.

For the perpendicular probe polarization, one needs to expand the angular parts of the $n p$ electron wave functions $Y_{1 m}(\theta, \phi)$ in terms of the spherical functions in a coordinate frame with the $z$ axis perpendicular to the original $z$ axis [17]:

$$
\begin{gathered}
Y_{11}(\theta, \phi)=\frac{1}{2} Y_{11}(\tilde{\theta}, \tilde{\phi})-\frac{1}{\sqrt{2}} Y_{10}(\tilde{\theta}, \tilde{\phi})+\frac{1}{2} Y_{1-1}(\tilde{\theta}, \tilde{\phi}), \\
Y_{10}(\theta, \phi)=\frac{1}{\sqrt{2}} Y_{11}(\tilde{\theta}, \tilde{\phi})-\frac{1}{\sqrt{2}} Y_{1-1}(\tilde{\theta}, \tilde{\phi}) \\
Y_{1-1}(\theta, \phi)=\frac{1}{2} Y_{11}(\tilde{\theta}, \tilde{\phi})+\frac{1}{\sqrt{2}} Y_{10}(\tilde{\theta}, \tilde{\phi})+\frac{1}{2} Y_{1-1}(\tilde{\theta}, \tilde{\phi})
\end{gathered}
$$

Here $\tilde{\theta}$ and $\tilde{\phi}$ are the polar angles of the new coordinate frame, obtained by rotation through 90 degrees about the original $y$ axis. These formulas show that for the states (A3) and (A4) with $M_{L}=0$, the average number of $m=0$ electrons detected in the perpendicular direction is unity, while for the states (A5)-(A7) with $M_{L}= \pm 1$ this number is 0.5 . Alternatively, one can expand the fine-structure states $|J, M\rangle$ in Eq. (1) in the frame with the perpendicular $z$ axis using equations similar to (A8)-(A10), and analyze the time evolution in it.
[1] L. Young, D. A. Arms, E. M. Dufresne, R. W. Dunford, D. L. Ederer, C. Höhr, E. P. Kanter, B. Krässig, E. C. Landahl, E. R. Peterson, J. Rudati, R. Santra, and S. H. Southworth, Phys. Rev. Lett. 97, 083601 (2006).

[2] N. Rohringer and R. Santra, Phys. Rev. A 79, 053402 (2009).

[3] M. A. Lysaght, P. G. Burke, and H. W. van der Hart, Phys. Rev. Lett. 102, 193001 (2009).

[4] L. Argenti and E. Lindroth, Phys. Rev. Lett. 105, 053002 (2010).
[5] E. Goulielmakis, Z.-H. Loh, A. Wirth, R. Santra, N. Rohringer, V. S. Yakovlev, S. Zherebtsov, T. Pfeifer, A. M. Azzeer, M. F. Kling, S. R. Leone, and F. Krausz, Nature (London) 466, 739 (2010).

[6] A. Fleischer, H. J. Wörner, L. Arissian, L. R. Liu, M. Meckel, A. Rippert, R. Dörner, D. M. Villeneuve, P. B. Corkum, and A. Staudte, Phys. Rev. Lett. 107, 113003 (2011).

[7] H. Hultgren, M. Eklund, D. Hanstorp, and I. Y. Kiyan, Phys. Rev. A 87, 031404(R) (2013). 
[8] M. Eklund, H. Hultgren, D. Hanstorp, and I. Y. Kiyan, Phys. Rev. A 88, 023423 (2013).

[9] H. F. Rey and H. W. van der Hart, Phys. Rev. A 90, 033402 (2014).

[10] A. Kramida, Yu. Ralchenko, J. Reader, and NIST ASD Team, NIST Atomic Spectra Database (ver. 5.3), [Online]. Available at http://physics.nist.gov/asd [2016, August 16]. National Institute of Standards and Technology, Gaithersburg, MD, 2015.

[11] G. F. Gribakin and M. Yu. Kuchiev, Phys. Rev. A 55, 3760 (1997).

[12] S. M. K. Law and G. F. Gribakin (unpublished).
[13] L. V. Keldysh, Zh. Eksp. Teor. Fiz. 47, 1945 (1964) [Sov. Phys. JETP 20, 1307 (1965)].

[14] S. F. C. Shearer, M. C. Smyth, and G. F. Gribakin, Phys. Rev. A 84, 033409 (2011).

[15] S. F. C. Shearer and M. R. Monteith, Phys. Rev. A 88, 033415 (2013).

[16] P. A. Korneev, S. V. Popruzhenko, S. P. Goreslavski, W. Becker, G. G. Paulus, B. Fetić, and D. B. Milošević, New J. Phys. 14, 055019 (2012).

[17] D. A. Varshalovich, A. N. Moskalev, and V. K. Khersonskii, Quantum Theory of Angular Momentum (World Scientific, Singapore, 1988). 